\title{
The first data on fungi and fungus-like organisms in Lake Baikal
}

Polyakova M.S. ${ }^{1 *}$, Mincheva E.V. ${ }^{2}$, Pudovkina T.A. ${ }^{2}$, Kabilov M.R. ${ }^{3}$, Tupikin A.E. ${ }^{3}$, Ishchenko A.A. ${ }^{1}$, Kurakov A.V. ${ }^{4}$, Sherbakov D.Yu. ${ }^{2,5}$

${ }^{1}$ Siberian Institute of Plant Physiology and Biochemistry SB RAS, Lermontov 132, Irkutsk, 664033, Russia

${ }^{2}$ Limnological Institute SB RAS, Ulan-Batorskaya 3, Irkutsk, 664033, Russia

${ }^{3}$ Institute of Chemical Biology and Fundamental Medicine SB RAS, Lavrentiev 8, Novosibirsk, 630090, Russia

${ }^{4}$ Moscow Lomonosov State University, Biological Faculty, Leninskie gory 1/12, Moscow, 119234, Russia

${ }^{5}$ Irkutsk State University, Karl Marks Str., 1, Irkutsk, 664003, Russia

\begin{abstract}
The first data on the taxonomic composition of the main groups of fungi and fungilike organisms in the grounds of coastal zone of Lake Baikal using metagenomic analysis based on the 18S rDNA (region V4) and ITS2 were obtained. Fungi were represented by five divisions: Ascomycota, Basidiomycota, Zoopagomycota, Chitridiomycota and Blastocladiomycota. There were also Cryptomycota, unclassified fungi and representatives of oomycetes and labyrinthulomycetes. Differences in the distribution of the main divisions of fungi in various sites of the lake were found. The creation of a unique collection of cultures of fungi and fungi-like organisms of Lake Baikal has begun which can be useful for further eco-physiological and biochemical studies.
\end{abstract}

Keywords: freshwater fungi, metagenome analysis, 18S rDNA (V4 region), ITS2, Lake Baikal

\section{Introduction}

The group of aquatic microscopic fungi remains almost unexplored in Lake Baikal. The species composition and functional role of fungi in the ecosystem of the lake are currently unknown. Fungi in aquatic ecosystems play important role is the decomposition of organic residues of various origin, in particular, such hardly biodegradable compounds as lignin and cellulose. They can live in the associations with algae, fishes and invertebrates with positive or negative influence to the hosts. Still there is no good data about isolation, morphological description and taxonomic identification of fungi from Lake Baikal. At the same time the recent publications (using high-throughput sequencing technology) allow us to assume the rather high diversity of fungi and fungi-like organisms in the lake (Kulakova et al., 2016; Yi et al., 2017; Mincheva et al., 2019). This study aimed to obtain the first data of main taxomonic groups of fungi inhabiting in the coastal zone of Lake Baikal.

\section{Material and methods}

Algae and their associated fungi were sampled taking into account the vertical zonation in the distribution of algae at ten sites of Lake Baikal with the assistance of scuba divers: Onokochanskaya Bay (a site with a massive growth of filamentous algae and visually clear), Irinda, Boro-Elga, Khara-Murin, Emelyanovka, Listvyanka, Kultuk, Baikalsk, and Maksimikha, at depths from 0.5 to $10 \mathrm{~m}$. DNA was extracted according to the modified Doyle and Dickson technique (Doyle and Dickson, 1987). Amplification and high-throughput sequencing for the 18S rDNA (V4 region) and ITS2 markers on the Illumina MiSeq platform were carried out at the SB RAS Genomics Core Facility (Novosibirsk, Russia). Contigs with $97 \%$ similarity were classified as a single operational taxonomic unit (OTU).

\section{Results}

Based on the ITS2 marker fungi in the studied depths of the lake were represented by five divisions: Ascomycota, Basidiomycota, Chytridiomycota, Zoopagomycota, and Blastocladiomycota. There were also unclassified fungi and representatives of Cryptomycota. Application of the marker also allowed to find the main groups of fungi and additionally the fungus-like organisms belonging to oomycetes and labyrinthulomycetes.

It was shown that there are significant differences on the composition of main taxonomic groups fungi and fungi-like organisms in the lake grounds of coastal zone 
from various sites. The largest number of sequences belonging to fungi and fungus-like organisms (according the ITS2 and 18S rDNA data) were detected in the lake grounds from the Kultuk and Khara-Murin sites. The lowest diversity of mycobiota were in the lake ground of Emelyanovka site, the members of Chytridiomycota and unclassified fungi-like groups only were detected.

\section{Discussion and conclusions}

The taxonomic composition of the main taxonomic divisions of fungi and fungi-like organisms were obtained in the lake ground of coastal zone of Baikal by using current molecular-genetic methods. Some differences in the distribution of these groups of organisms in the various locations were discovered. We can suggest that their role in Baikal ecosystem is versatile.

In aquatic ecosystems, basidiomycetes are very poorly represented. Most of the detected basidiomycetes are likely invasive from land and are not truly aquatic fungi.

Chytrid fungi comprised the maximum proportion in the community from the Khara-Murin site. Chytrid fungi are parasites of various aquatic organisms or are saprotrophs that live on the remains of plant and animal origin that have fallen into the water. Moreover, recent studies of Gerphagnon et al. (2013) indicated that chytrid fungi can be phytoplankton parasites and have a suppressive effect on the intensity of cyanobacterial blooms.

Members of Zoopagomycota were detected only in the communities from Baikalsk and Listvyanka (the sites are under anthropogenic impact). Members of this division are obligate parasites of other fungi and microscopic animals (nematodes, rotifers and amoebas).

Within the division, Ascomycetes account for 60 thousand species; however, in aquatic biotopes, only several hundreds of the species representing this class are known. Many aquatic representatives of Ascomycota belong to saprotrophs and there are also many parasitic fungi among them (mainly on algae, less often on animals). By plating method on nutrient media high diversity of these taxonomic groups of fungi were detected.
The members of Blastocladiomycetes discovered in the lake ground from the Kultuk and KharaMurin sites that rich by organic matter. In Kultuk, the accumulation of vegetation is due to weakened hydrodynamics, and in Khara-Murin - the influence of the river runoff is going.

Using the traditional methods of cultivation, we initiated the creation of the unique collection of Baikal fungi and fungus-like organisms, which is stored at Siberian Institute of Plant Physiology and Biochemistry Siberian Branch of the Russian Academy of Sciences and Department of Mycology and Algology of Biological Faculty of Moscow Lomonosov State University.

\section{Acknowledgements}

We thank I.V. Khanaev, I.A. Nebesnykh and V.I. Chernykh for their assistance in sampling. This study was supported by project No. 03452019-0004 (AAAA-A16-116122110060-9) and № AAAA-A16-116021660088-9 funded by the Government, and by project No. 17-44-388071r_a funded by RFBR and the Government of the Irkutsk Region.

\section{References}

Doyle J.J., Dickson E.E. 1987. Preservation of plant samples for DNA restriction endonuclease analysis. Taxon 36(4): 715-722. DOI: $10.2307 / 1221122$

Gerphagnon M., Latour D., Colombet J. et al. 2013. Fungal parasitism: life cycle, dynamics and impact on cyanobacterial blooms. PloS one 8(4). DOI: 10.1371/journal.pone.0060894

Kulakova N.V., Adelshin R.V., Khanaev I.V. 2016. Metagenomic analysis of the community of bacterial and eukaryotic communities in normal and diseased Lubomirskiidae sponges. Vestnik Nauchnykh Konferentsiy [Bulletin of Scientific Conferences] 12: 28. (in Russian)

Mincheva E.V., Peretolchina T.E., Kravtsova L.S. et al. 2019. Hidden diversity of micro-eukaryotes in Lake Baikal: a metagenomic approach. Limnology and Freshwater Biology 1: 150-154. DOI: 10.31951/2658-3518-2019-A-1-150

Yi Z., Berney C., Hartikainen H. et al. 2017. High throughput sequencing of microbial eukaryotes in Lake Baikal reveals ecologically differentiated communities and novel evolutionary radiations. FEMS Microbiology Ecology 93. DOI: $10.1093 /$ femsec/fix073 